\title{
Oral Mucositis, Pain and Xerostomia in 135 Head and Neck Cancer Patients Receiving Radiotherapy with or without Chemotherapy
}

\author{
Ourania Nicolatou-Galitis ${ }^{*}, 1$, Vassilis Kouloulias ${ }^{2}$, Anastasia Sotiropoulou-Lountou ${ }^{3}$, \\ Konstantinos Dardoufas ${ }^{4}$, Argyro Polychronopoulou ${ }^{5}$, Pavlina Athanassiadou ${ }^{6}$, \\ Georgia Kolitsi ${ }^{4}$ and John Kouvaris ${ }^{7}$
}

\author{
${ }^{1}$ Dental Oncology Unit, Clinic of Hospital Dentistry, School Dentistry, University of Athens, Thivon 2, Goudi, 115 27, \\ Athens, Greece \\ ${ }^{2}$ Department of Radiotherapy, Attikon University Hospital, Rimini 1, Haidari, 124 64, Athens, Greece \\ ${ }^{3} 2^{\text {nd }}$ Radiothepapy Department, Saint Savvas Anticancer Hospital, Alexandras Avenue 171, 115 22, Athens, Greece \\ ${ }^{4}$ Radiation Oncology Department, Hygeia Hospital, Kifissias Avenue and Erythrou Stavrou 4, Maroussi, 151 23, Athens, \\ Greece \\ ${ }^{5}$ Department of Preventive and Community Dentistry, School of Dentistry, University of Athens, Thivon 2, Goudi, 115 \\ 27, Athens, Greece \\ ${ }^{6}$ Department of Pathologic Anatomy, School of Medicine, University of Athens, Mikras Asias 75, Goudi, Athens, Greece \\ ${ }^{7}$ Department of Radiotherapy, Aretaieion University Hospital, School of Medicine, University of Athens, Vas Sofias 76, \\ 11528 Athens, Greece
}

\begin{abstract}
Goal of work: This study compared the severity of oral mucositis, pain and xerostomia during and at the completion of radiotherapy in head and neck cancer patients who had received antifungal and antiviral treatment. Patients: The study included 135 patients. Mean total radiotherapy dose was 62.4 Gray. Chemotherapy was administered to $47 \%$ of patients. Methods: Oral mucositis was scored weekly, while patients self-evaluated their pain and xerostomia. Cytology smears for the assessment of herpetic infection complicating the ulcers of mucositis were taken from 46 patients. Systemic antifungals and antivirals were administered during radiotherapy, upon clinical, presumptive diagnosis of candidiasis and herpetic infection. Antifungals and antivirals were continued to the end of radiotherapy. Results: Radiotherapy was completed within the preplanned time in 117 patients $(87 \%)$. During radiotherapy, the prevalence of severe mucositis, pain and xerostomia was $57 \%, 43 \%$ and $29 \%$ respectively, and was significantly reduced to $33 \%,(P<0.001)$, to $24 \%$, $(P<0.001)$, and to $18 \%,(P<0.05)$ at the end of radiotherapy. Antifungals and antivirals were utilized in $70 \%$ and $71 \%$ of patients, respectively. Viral cytology was positive in 14 of $46(30.4 \%)$ patients. Conclusions: The significant reduction of severe oral mucositis, pain and xerostomia at time of completion of radiotherapy, as compared to during the course of radiotherapy, after the treatment and prevention of candidiasis and herpes, denotes an important role of these infections in radiation-induced mucositis. Limitations of the study are the practical issues of the lack of the verification of the fungal status before and after treatment and of the verification of the viral status in only $47.9 \%$ (46 of 96) of the patients with a clinical suspicion of herpetic infection. A controlled study is needed to investigate and further clarify the role of antifungal and antiviral prophylaxis relative to oral mucositis, pain and xerostomia during head and neck RT.
\end{abstract}

Keywords: Head and neck radiotherapy, oral mucositis, pain, xerostomia, antifungal and antiviral treatment.

\section{INTRODUCTION}

Oral mucositis can be the most debilitating and troublesome side effect in patients with head and neck cancer, who receive radiotherapy (RT), with or without chemotherapy. It is associated with considerable pain, which can significantly impair quality of life. The severity of oral mucositis increases over time, during the course of RT and patients report

*Address correspondence to this author at the Bouboulinas 41, N. Psyhico, 154 51, Athens, Greece; Tel: +30 69446015 29; Fax: +30 2106775 567; E-mail: nicolatou.galitis@1ycos.com significant worsening of pain, xerostomia and other related oral symptoms at the end as compared to the start of RT [15]. Mucositis occurs among virtually all patients who are undergoing radiation or chemoradiation therapy for head and neck cancers. In several reports, $35 \%$ to over $60 \%$ of patients develop severe mucositis, which is not relieved by opioid analgesia. Radiation-induced oral mucositis has also a significant economic impact due to costs associated with pain management, liquid diet supplements, gastrostomy tube placement or total parenteral nutrition, management of secondary infections and hospitalizations [6, 7]. Furthermore, patients with severe mucositis are at higher risk of unplanned 
breaks/delays in radiation therapy, adversely affecting tumor control and treatment outcomes [8-10].

Mucosal erythema (mucositis grade 1) is followed by foci of ulceration (mucositis grade 2) which becomes confluent over time (severe mucositis grade 3 and 4). A fibrinous exudate that is colonized by bacteria and covers the ulcer typically develops; this component of the lesion is referred to as a "pseudomembrane". Mucositis is not an infectious process per se, but fungal, viral, and/or bacterial infections may co-exist with the mucositis and further exacerbate pain. Appropriate diagnosis and treatment of oral infections are important [11-16]. Although studies on bacterial infections complicating oral mucositis are inconclusive [17-20], candidiasis and herpes simplex virus- 1 infection have been reported by several investigators to develop and be superimposed on radiation mucositis [21-24]. Oral candidiasis and herpes simplex virus-1 infection, presenting as erythematous, pseudomembranous or ulcerative lesions, may be mistaken for oral mucositis and must be differentially diagnosed and treated with appropriate antifungal and antiviral medications [14-16]. The differential diagnosis of oral mucositis from infections is, however, difficult or at times impossible [14, 15, 21-23].

Oral pseudomembranous candidiasis develops with an incidence of $27 \%$ to $52.5 \%$ in head and neck radiation patients and is often superimposed on radiation-induced ulcerative/pseudomembranous grade 2, 3, or 4 mucositis. Candidiasis may recur during the course of RT [21-26].

Oral candidiasis has been related to xerostomia [21, 24, 26]. Epstein et al. [21] and Nicolatou-Galitis et al. [26] reported that radiation-induced xerostomia correlates with risk for oropharyngeal candidiasis.

On the other hand, Nicolatou-Galitis et al. [24] reported that sudden onset or the worsening of xerostomia in head and neck cancer patients receiving RT was a presenting symptom for candidiasis.

Candidiasis develops in a Candidia carrier. The reported prevalence of Candida carriers during head and neck radiotherapy ranges between $57 \%$ to $70.6 \%$ [25, 27, 28]. In our patients at our institution candidiasis has been diagnosed with a prevalence between $34.5 \%$ to $50 \%$, while Candida carriage has been confirmed in $62 \%$ to $70.6 \%$ of the patients [22-24, 26].

Antifungal prophylaxis has been utilized by some clinicians due to the high incidence of candidiasis, risk of recurrences and difficulties in establishing a differential diagnosis. Administration of systemic antifungal prophylaxis, either from the initiation $[22,23,29]$ or one week after the start of RT [30], was shown to have a beneficial effect on the severity of oral mucositis and RT interruptions.

Herpes simplex virus-1 infection has also been shown to complicate oral mucositis in head and neck RT as well. In our Clinic, herpes virus was cultured from the ulcers of 5 of 14 patients [24], while it was identified in 14 out of 29 available smears among 48 patients with ulcerative mucositis (29\%) [23]. The majority of our patients with positive viral cytology responded to antiviral treatment: a reduction of the grade of oral ulcerative mucositis was subsequently noted.
Herpes simplex reactivation both before and after cancer therapy has been reported to be extremely frequent [31]. Patients, found HSV positive with direct immunofluorescence, seemed to have more severe oral mucositis than HSV negative patients, while a positive IgM result was more frequent in the mucositis group of patients.

Since 2005 systemic antifungals and antivirals have been administered to our head and neck cancer patients during RT when a candidal or herpetic infection is clinically diagnosed; this approach has been based on published clinical criteria [22-24]. The administration of antifungals and/or antivirals is continued to the end of radiotherapy to prevent recurrences. The above clinical policy has been based on (1) high prevalence of Candida carriage and candidiasis and the risk of recurrences [22, 24-28], (2) incidence of HSV-1 reactivation and infection [23, 24, 31], (3) the reported beneficial effect of systemic antifungal and antiviral medication [22-24, 29, 30], and (4) difficulties in delineating differential diagnosis of infections from mucositis [14, 15, 21].

The purpose of this study is to compare the severity of oral mucositis, pain and xerostomia during and at the completion of radiotherapy in head and neck cancer patients who had received antifungal and antiviral treatment.

Our hypothesis is that if candidiasis and/or herpetic infection exacerbate mucositis, pain and xerostomia then their treatment, followed by infection prevention throughout the course of RT should reduce the severity of oral mucositis, pain and xerostomia.

\section{PATIENTS AND METHODS}

\section{Patients, Eligibility Criteria and Radiotherapy Characteristics}

One hundred and thirty five out of 230 consecutive patients, with head and neck cancer, eligible to receive radiotherapy, who were referred to our Clinic for routine oral oncology supportive care, were followed weekly until the end of RT and were included in the study. The rest 95 patients did not comply with the requested weekly follow up and were not evaluated.

Patient demographics, tumor type and radiotherapy characteristics are shown in Table $\mathbf{1}$.

General blood tests and liver and renal function were within normal limits. Karnofsky performance status ranged between 80 and $100 \%$.

Patients were irradiated with a 6-MV linear accelerator.

Concomitant chemotherapy of cisplatinum, $75 \mathrm{mg} / \mathrm{m}^{2}$ every 21 days was administered to 55 patients. Nine patients received cisplatinum, as above and cetuximab, at an initial dose of $400 \mathrm{mg} / \mathrm{m}^{2}$, followed by $250 \mathrm{mg} / \mathrm{m}^{2}$ every two weeks.

All patients were thoroughly informed about their disease and the treatment they would receive. All patients provided informed consent.

\section{Oral Evaluation and Oral Supportive Oncology Care}

The oral cavity was assessed in a dental office setting outside the context of a clinical trial, in consecutive patients, 
Table 1. Patient Demographics, Tumor Type, Dose of RT. (N=135)

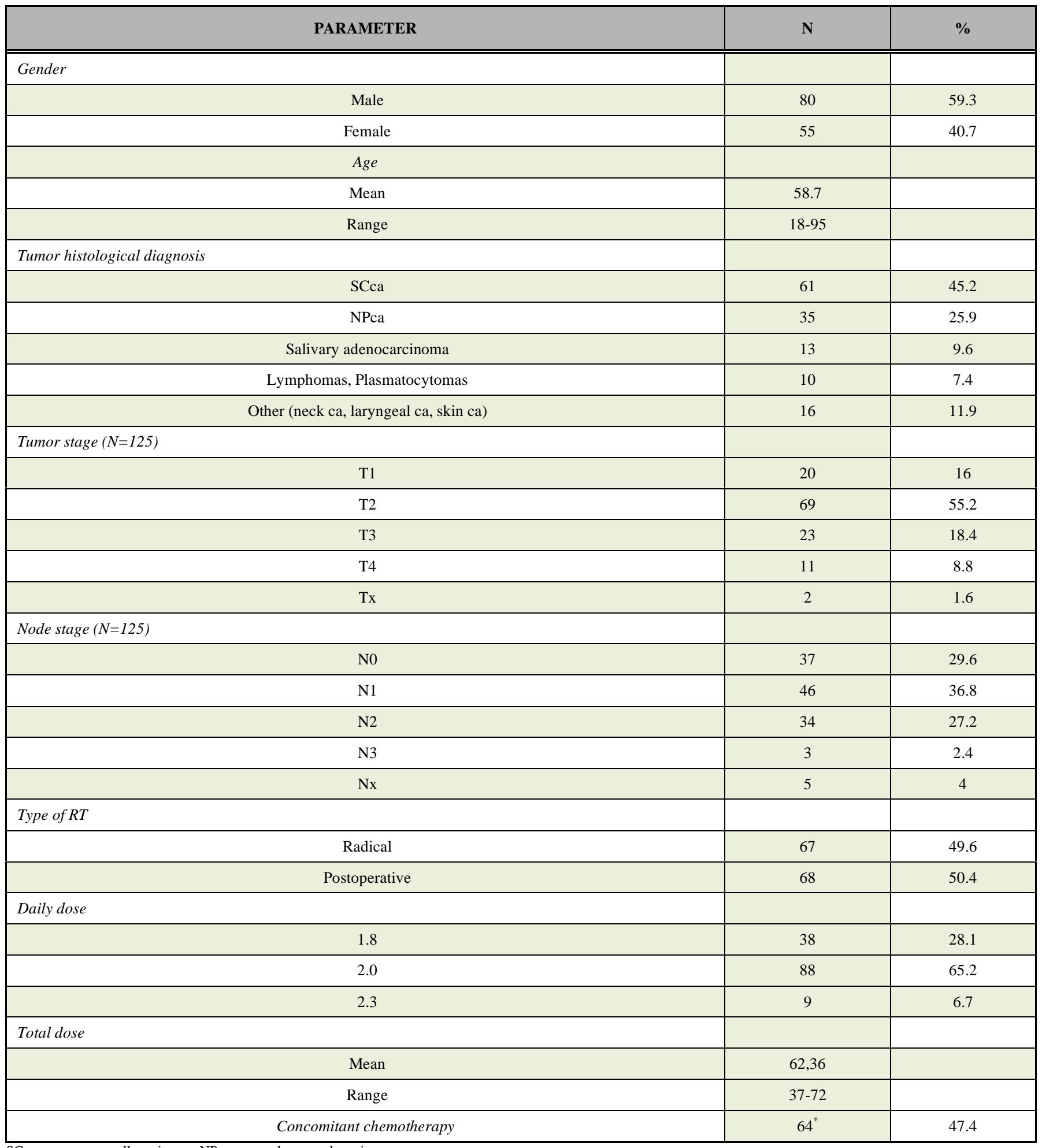

SCca $=$ squamous cell carcinoma, NPca $=$ nasopharyngeal carcinoma

Stage of lymphomas: IIE $=6$ cases, IE $=4$ cases

*30 of those 64 patients were also included in our paper, reference [33].

referred from the Cancer Centers to our Dental Oncology Clinic, for routine oral oncology supportive care. Patients were examined weekly or more often, upon their request. Standard oral mucosal and dental care was introduced to all patients. Coating and mucosal moisturizing agents and topical anesthetics were also introduced.
Oral mucositis was recorded according to EORTC/ RTOG criteria [32], (Figs. 1 and 2), as follows: Grade 1 (diffuse erythema, patient can eat solid food), Grade 2 (erythema and small foci of ulcers, patient can take soft diet), Grade 3 (painful ulcers extending on more than half of the oral mucosa, patient can take liquids only), Grade 4 (painful 


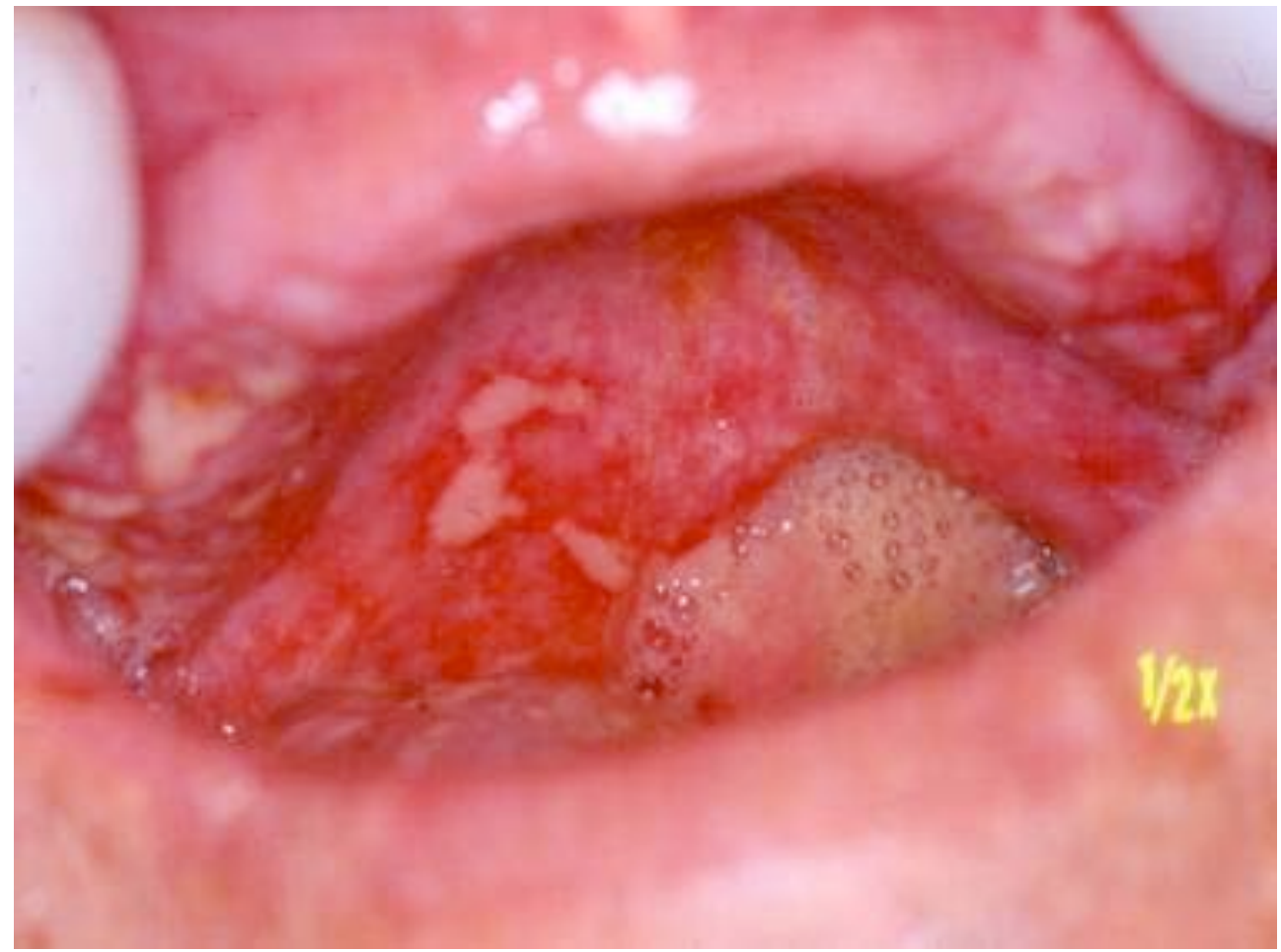

Fig. (1). Radiation-induced mucositis grade 3 on hard palate (palatal ca). Pseudomembranes cover the painful ulcerations.

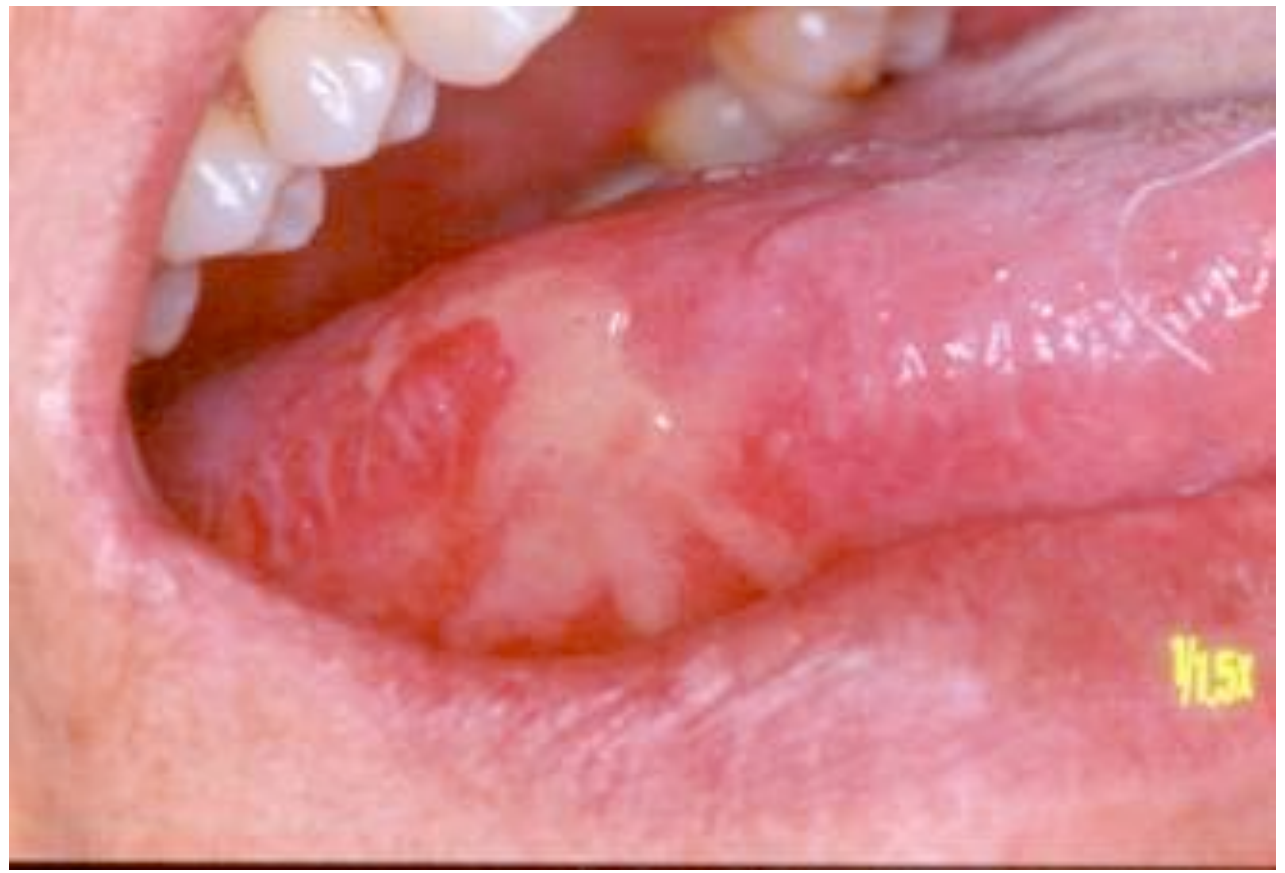

Fig. (2). Radiation-induced mucositis grade 3 on the lateral border of the tongue (tongue ca). Pseudomembranes cover the painful ulcerations.

ulcers covering almost all mucosal surfaces, alimentation is not possible).

A presumptive diagnosis of oral candidiasis and herpes simplex virus-1 infection was made upon clinical suspicion. Erythema located on the central dorsum of the tongue, or on the central area of the hard palate, or bilateral, symmetrical erythema of the buccal mucosa, and angular cheilitis and non painful easily removable whitish pseudomembranes were indicative of the different clinical forms of candidiasis (Figs. 3 and 4). A systemic antifungal medication (itraconazole, fluconazole or posaconazole) was administered for 1 or two weeks, as introduced by the manufacturer and continued as prophylaxis to the end of RT. Nine patients received systemic antifungal prophylaxis from the initiation of RT, per request of his/her oncologist. 


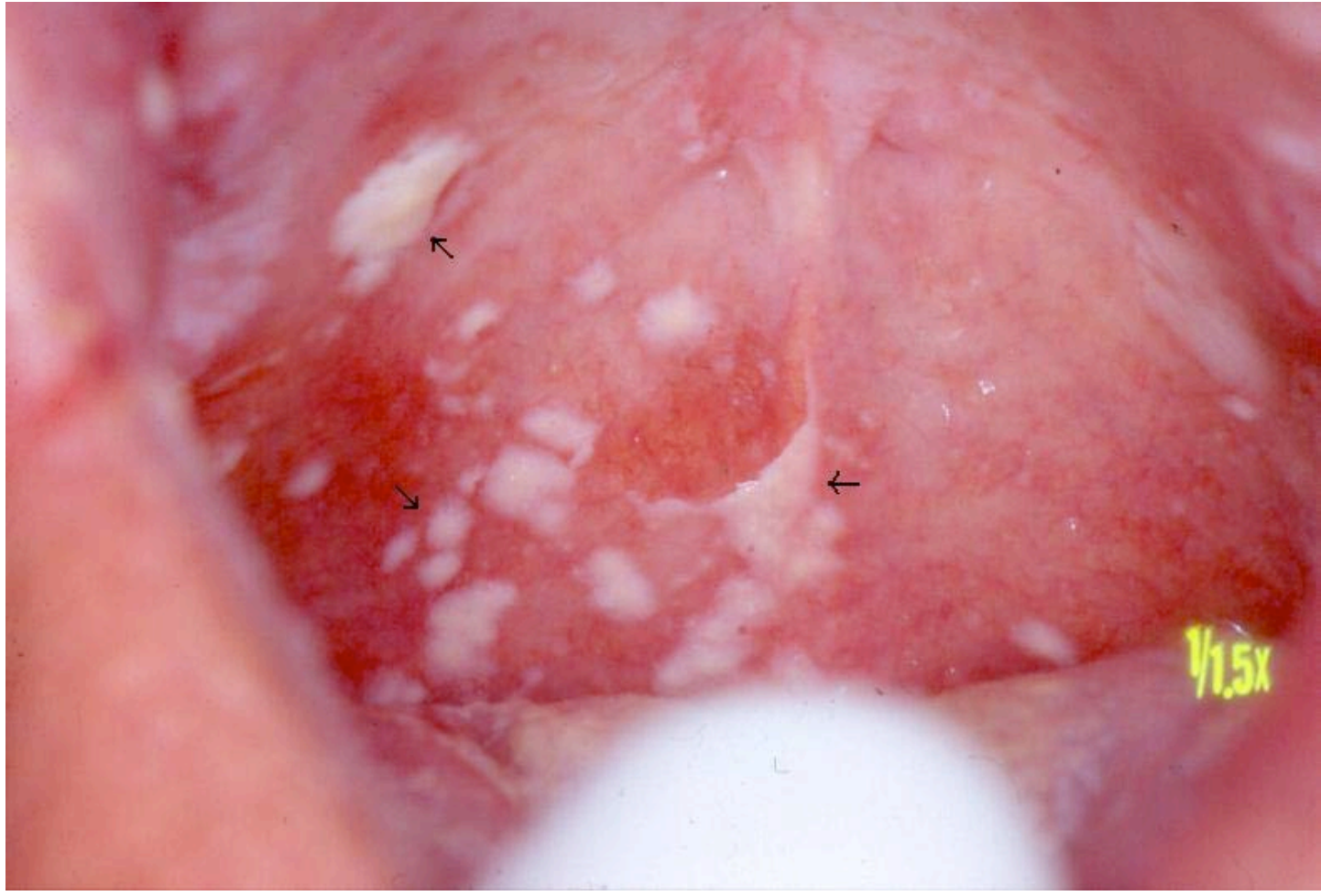

Fig. (3). Pseudomembranous candidiasis on hard palate during RT (ca, floor of mouth). Easily removable, non-painful pseudomembranes are seen on the palatal mucosa. Smear was positive for Candida, while lesion healed with systemic antifungal treatment.

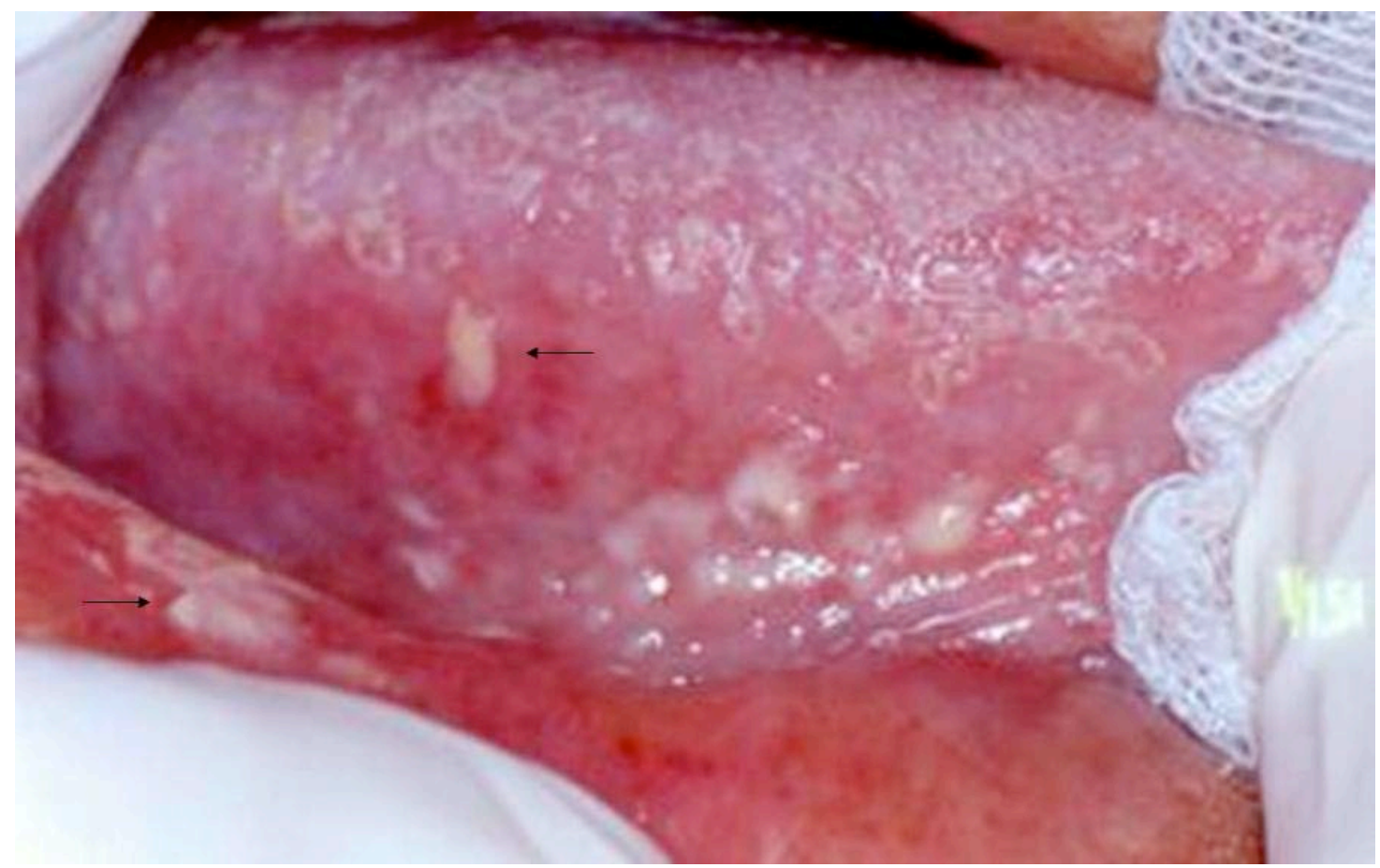

Fig. (4). Pseudomembranous candidiasis on the lateral border of the tongue during RT (ca, floor of mouth). Easily removable, non-painful pseudomembranes are seen on the tongue mucosa. Smear was positive for Candida, while lesion healed with systemic antifungal treatment.

Herpes labialis, ulcers coalescing on the dorsum of the tongue or on the hard palate, early initiation of ulcerative mucositis, or sudden development or worsening of mucositis were viewed as indicative of herpetic infection (Figs. 5, 6 and 7). A systemic antiviral medication (acyclovir or vala- cyclovir), as indicated by the manufacturer, was administered and continued as prophylaxis to the end of RT. Cytology smears were taken from 46 patients and were stained using Papanicolaou stain to assess the herpetic infection. 


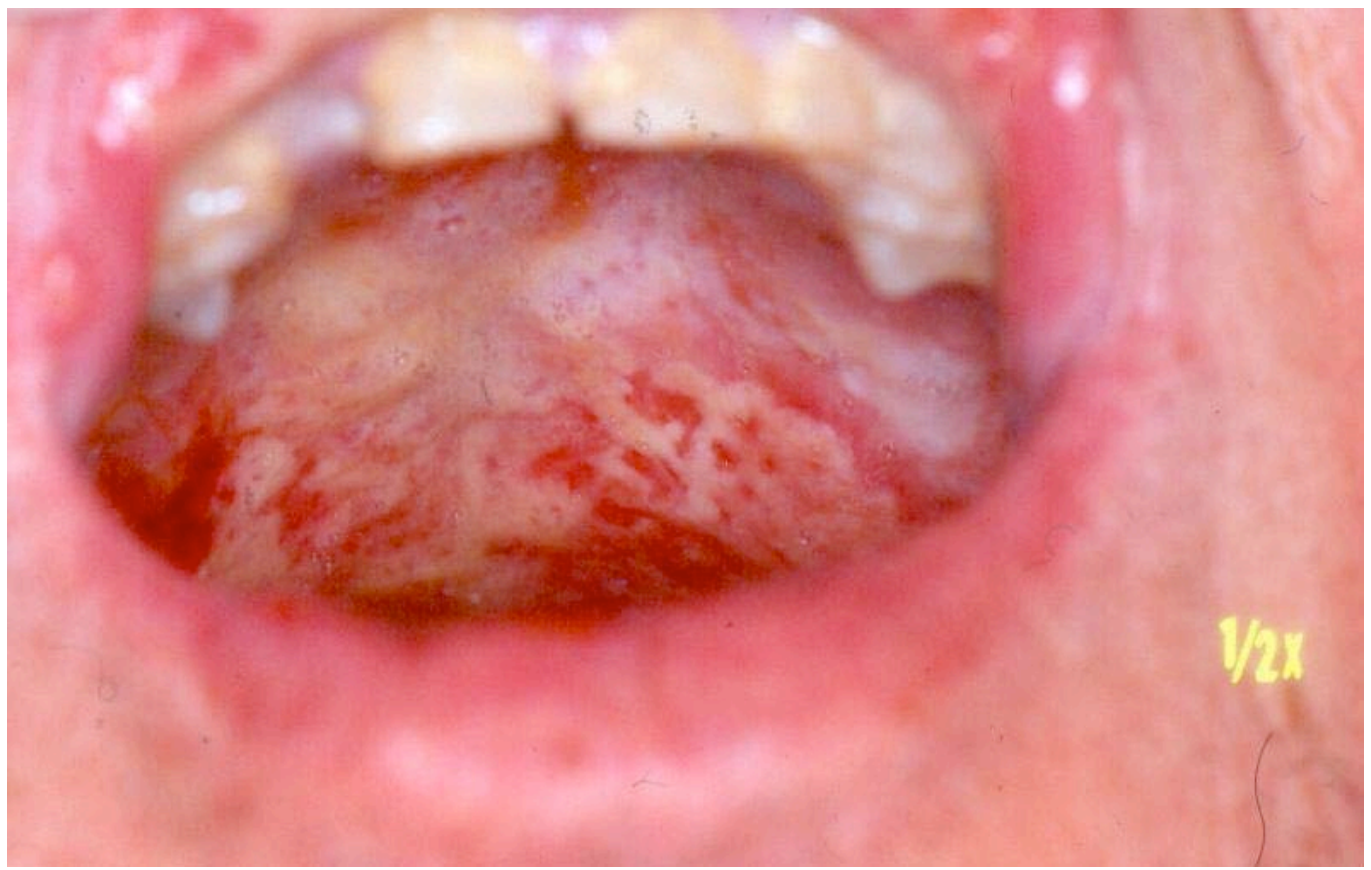

Fig. (5). Painful, non-healing ulcerations, covered by pseudomembranes, diagnosed as mucositis grade 4, after the completion of RT (ethmoid ca). Smear showed viral cytopathic alterations, while patient responded to acyclovir. Pseudomembranes cover the painful ulcerations.

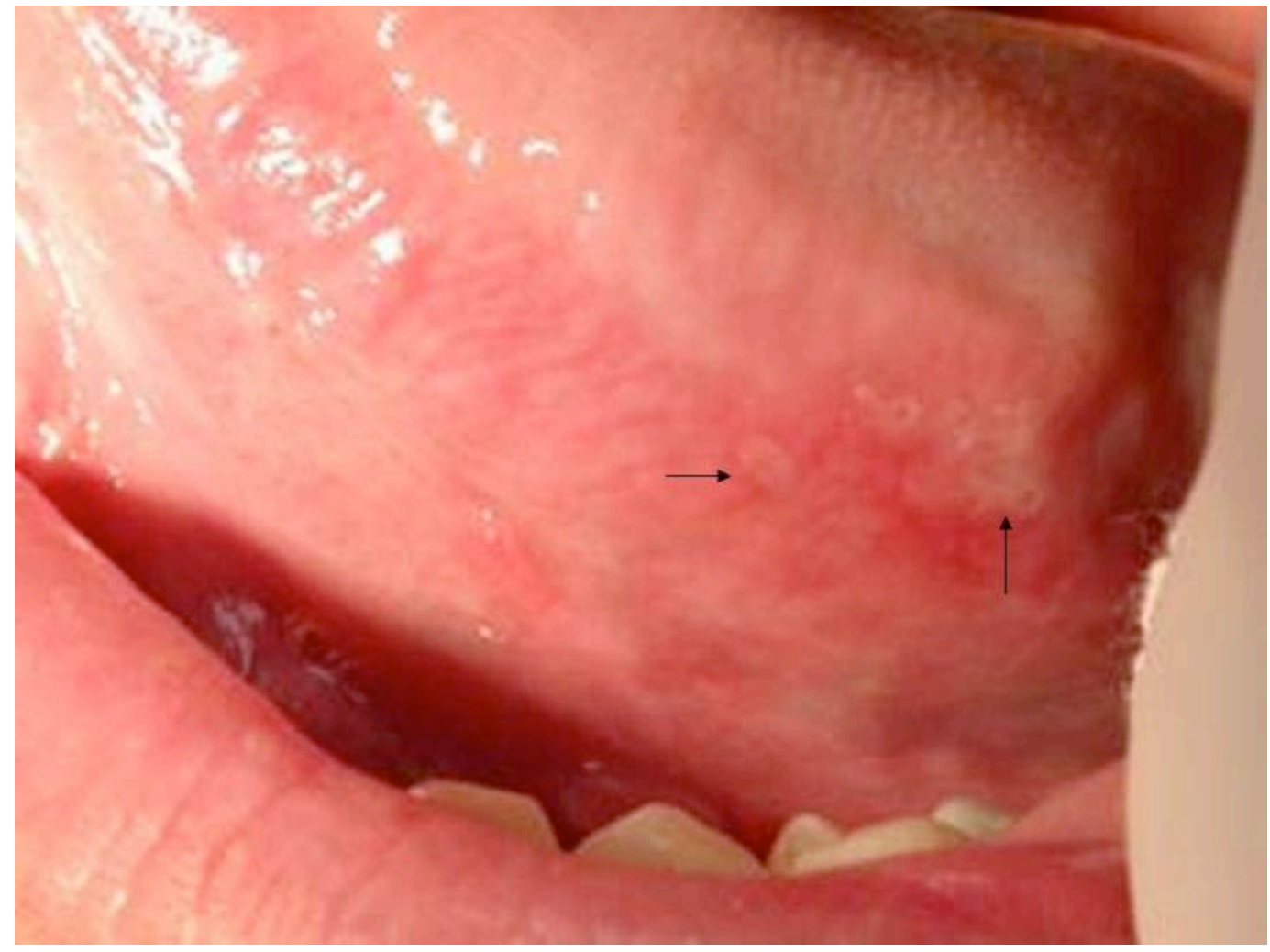

Fig. (6). Painful ulcerations, covered by pseudomembranes, scored as mucositis grade 2, at the $30^{\text {th }}$ fraction of RT (nasopharyngeal ca). The patient had not developed mucositis throughout RT until that day. The differential diagnosis included herpes simplex virus infection. Smear verified HSV-1 infection. Ulcers healed after the administration of acyclovir.

\section{Oral Pain and Xerostomia}

Oral pain and xerostomia were evaluated by the patients, using a $10 \mathrm{~cm}$ visual analogue scale with scores of 0 to 10 , where 0 was no pain or xerostomia respectively and 10 was highest pain or xerostomia. Scores between 1 to 4 were considered as mild, scores between 5 to 7 as moderate and 8 


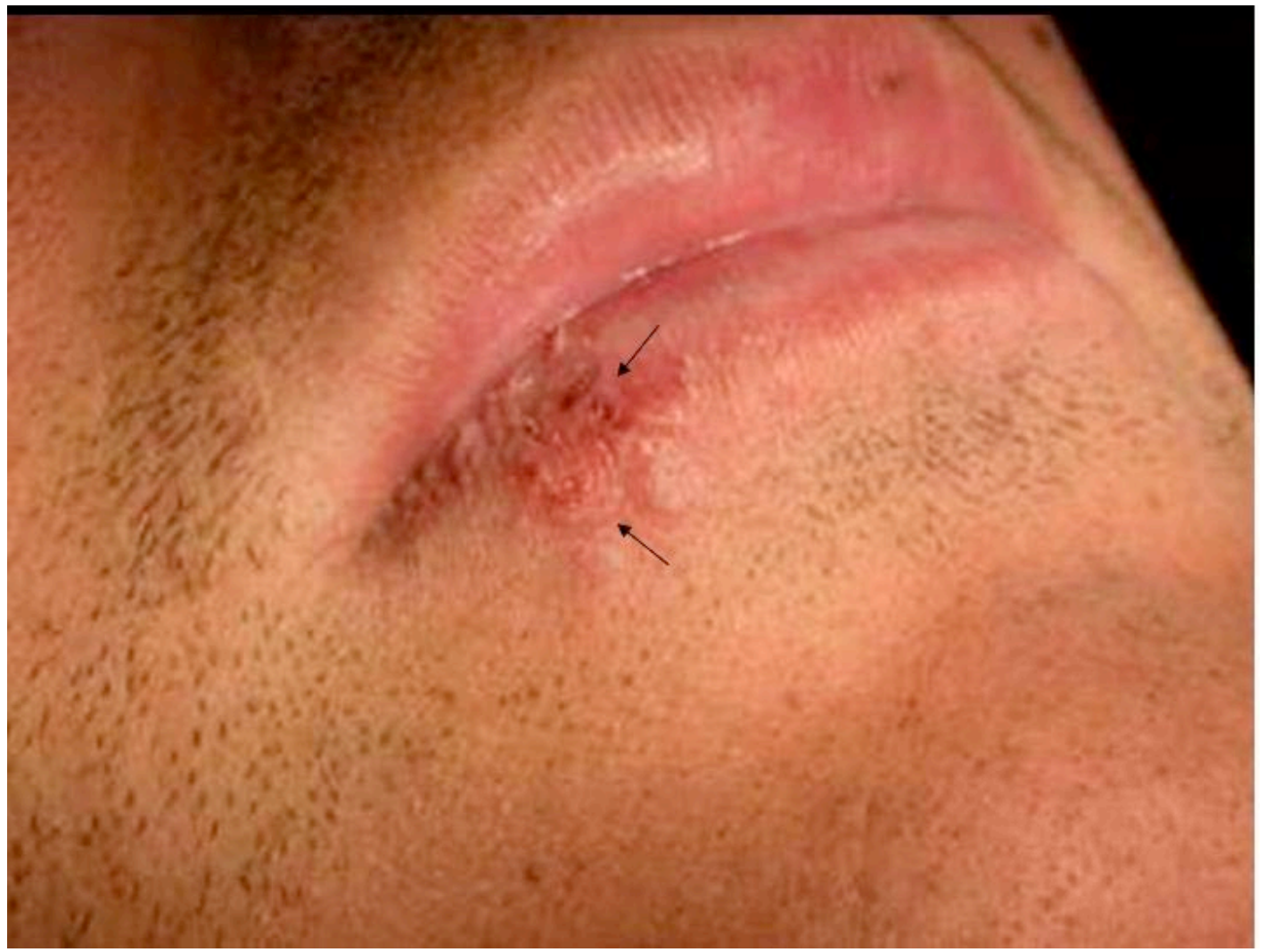

Fig. (7). The same patient of Fig. (6). Herpes labialis is seen, concomitantly with the tongue ulcerations and further led to the suspicion of herpes.

to 10 were considered as very much or severe, as the patients commented. Patients were asked to score their "average, within the day", pain and xerostomia. Data regarding pain medication use administered by the radiotherapists or medications with a side effect of xerostomia were not collected. No patient received pilocarpine.

\section{Statistical Analysis}

Standard statistical methods were used for data description. Statistical analysis relied on McNemar's chi square test or Pearson's chi square test depending on the nature of examined variables. Data were analyzed using the STATA® 10.0 statistical package. All statistical tests were two sided, and level of statistical significance was set at $5 \%$.

\section{RESULTS}

\section{Radiotherapy}

Of all 135 patients, 54 were referred for oral care before the initiation of radiotherapy and 81 patients within the first week, after the initiation of radiotherapy.

Eighteen patients $(13.3 \%)$ interrupted treatment; twelve $(8.8 \%)$ due to severe mucositis and six patients $(4.5 \%)$ due to fever and fatigue (1 patient), dermatitis (3 patients), and myelotoxicity (2 patients). Two patients (1.4\%) received nasogastric tube and four patients $(2.8 \%)$ were hospitalized.

Weight changes were available in 101 patients, as shown in Table 2.

\section{Symptom Burden during the Course of RT}

\section{Oral Mucositis, Pain and Xerostomia}

Oral ulcerative mucositis grade 2, 3, and 4 was observed in 111 of all 135 patients $(82 \%)$. Ulcerations were initiated at a mean of 14.6 RT fractions (sd: 5.2, range: 3-30). Mild to moderate mucositis grade 1 and 2 was evaluated in 58 patients, while severe mucositis grade 3 and 4 was scored in 77 patients, as shown in Table 2.

Oral pain and xerostomia were available from 131 patients, also shown in Table 2.

Severe xerostomia was significantly increased in patients who received antifungals as compared to the ones who did not receive antifungals (chi square test, $P<0.05$ ), while it was not significantly increased in association with use of antivirals.

\section{Overall Symptom Burden}

Forty-three patients developed only mild to moderate symptoms. Severe oral mucositis, severe pain and severe xerostomia were simultaneously recorded and /or reported in 22 patients. Different numbers of patients developed / reported one or two severe symptoms at the same time.

No significant differences in symptom burden were found between patients who received radiotherapy alone as opposed to patients who received radiotherapy with concomitant chemotherapy. 
Table 2. Oral Mucositis, Pain and Xerostomia during and after RT $(n=135)$

\begin{tabular}{|c|c|c|c|c|c|}
\hline \multirow{2}{*}{ PARAMETER } & \multicolumn{2}{|c|}{ During RT } & \multicolumn{2}{|c|}{ After RT } & \multirow{2}{*}{$\begin{array}{c}p \text {-value } \\
\text { McNemar's } X^{2}\end{array}$} \\
\hline & $\mathbf{N}$ & $\%$ & $\mathbf{N}$ & $\%$ & \\
\hline \multicolumn{6}{|c|}{ Mucositis } \\
\hline Mild/moderate, grade $\leq 2$ & 58 & 43.0 & 90 & 66.7 & \multirow{2}{*}{$<0.001$} \\
\hline Severe, grade 3,4 & 77 & 57.0 & 45 & 33.3 & \\
\hline \multicolumn{6}{|c|}{ Pain $(n=131)$} \\
\hline Mild/moderate & 75 & 57.2 & 99 & 75.6 & \multirow{2}{*}{$<0.001$} \\
\hline Severe & 56 & 42.8 & 32 & 24.4 & \\
\hline \multicolumn{5}{|c|}{ Xerostomia $(n=131)$} & \\
\hline Mild/moderate & 93 & 71.0 & 107 & 81.7 & \multirow{2}{*}{$<0.05$} \\
\hline Severe & 38 & 29.0 & 24 & 18.3 & \\
\hline Use of antifungals & 95 & 70.3 & & & \\
\hline Use of antivirals & 96 & 71 & & & \\
\hline Cytology smear positive $(n=46)$ & 14 & 30.4 & & & \\
\hline \multicolumn{5}{|c|}{$R T$ interruptions } & \\
\hline Due to mucositis & 12 & 8.8 & & & \\
\hline Other reasons & $6^{*}$ & 4.5 & & & \\
\hline \multicolumn{5}{|c|}{ Weight, $k g(n=101)$} & \\
\hline \multicolumn{6}{|l|}{$\operatorname{Loss}(n=81)$} \\
\hline Mean & 5.6 & & & & \\
\hline Range & $1-21$ & & & & \\
\hline \multicolumn{6}{|l|}{$\operatorname{Gain}(n=3)$} \\
\hline Mean & 3.7 & & & & \\
\hline Range & $3-4$ & & & & \\
\hline \multicolumn{6}{|l|}{ No change $(n=17)$} \\
\hline 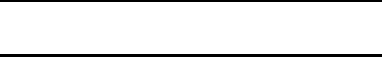 & & & & & \\
\hline
\end{tabular}

\section{Antifungals and Antivirals}

Systemic antifungal and antiviral medication was administered to 95 patients (70.3\%) and to 96 patients (71\%) respectively.

Cytology smear for viral verification was obtained from 46 of 96 patients $(46 / 96,47.9 \%)$, who received antiviral medication, after the clinical suspicion of herpes.

Fourteen cytology smears, taken from ulcerative lesions of 46 patients $(14 / 46,30.4 \%)$ showed microscopic cytopathic alterations indicative of the presence of herpetic infection. Five of the 14 viral-positive patients (35.7\%) were receiving concomitant chemotherapy. One patient did not respond to antivirals and had interrupted RT, 3 patients remained stable and 10 patients showed a reduction in the grade of mucositis and pain severity.

\section{Symptom Burden at the End of RT}

\section{Oral Mucositis, Pain and Xerostomia}

Overall ulcerative mucositis grade 2, 3 and 4 was evaluated in 105 patients (77.7\%), thus, remaining as high as it was 
evaluated during the course of RT. Prevalence of severe mucositis, severe pain and severe xerostomia were significantly reduced to $33.3 \%(P<0.001)$, to $24.4 \%(P<0.001)$ and to $18.3 \%(P<0.05)$, respectively, as shown in Table 2.

Xerostomia was not significantly increased in patients who received antifungals or antivirals as compared to patients who did not receive antifungals or antivirals.

\section{Overall Symptom Burden}

Seventy-four patients completed RT with only mild to moderate symptoms, while 10 patients completed RT having simultaneously three severe symptoms.

These differences of symptom burden during and at the end of RT were statistically significant by Pearson chi square analysis $P<0.001$.

\section{Radiotherapy alone versus Chemoradiotherapy}

No significant differences in symptom burden were found between patients who received radiotherapy alone as opposed to patients who received radiotherapy with concomitant chemotherapy.

\section{Follow - Up}

No significant differences in the follow - up data were observed in patients with or without RT interruptions, as shown in Table $\mathbf{3}$.

\section{DISCUSSION}

One hundred and thirty-five head and neck cancer patients who received radiotherapy with or without chemotherapy were followed prospectively from the initiation to the end of RT, with respect to oral mucositis, pain, and xerostomia. Antifungal and antiviral treatment, throughout the course of radiotherapy, were administered to $70 \%$ and $71 \%$ of the patients, respectively.

Significant reduction of the severity of oral mucositis, pain, and xerostomia was observed at the end of RT, as compared to that assessed during the course of RT. Those results are in agreement with our hypothesis and delineate an important role of fungal and viral infection in the severity of oral mucositis, pain and xerostomia.

It is not clear in the present patient cohort, however, which or to what extent, antifungal and/or antiviral treatment affected the severity of radiation- and / or chemoradiotherapy- induced oral mucositis and its associated pain. The significant reduction of severe xerostomia in patients who received antifungals denotes that the development of candidiasis may increase the severity of radiation-induced xerostomia. Our group has reported a beneficial effect of administration of antifungal and antiviral medication on severity of oral mucositis and its associated pain [22-24, 33]. Other investigators have reported similar results as well [29, 30]. However, limitations in the study design were present, such as the non-randomized patient groups, or the use of historical controls, or the lack of (a) the description of the grading / scoring system of mucositis or (b) of the criteria for the differential diagnosis of mucositis from infections and the lack of laboratory verification of infections.

Xerostomia has been related to candidiasis in previous reports, too [21, 24, 26]. As it is well known, head and neck radiotherapy is a major cause of xerostomia. Within this context, Epstein et al. [21] and Nicolatou-Galitis et al. [26] have reported that radiation-induced xerostomia in head and neck cancer patients who received RT correlates with risk for oropharyngeal candidiasis. On the other hand, NicolatouGalitis et al. [24] reported that sudden onset or the worsening of xerostomia in head and neck cancer patients receiving $\mathrm{RT}$ was a presenting symptom for candidiasis.

In the present report the significant reduction of xerostomia at the end of RT, which was found significantly associated with the antifungal medication, denoted that severe xerostomia may be a presenting symptom of candidiasis. This finding was consistent with our prior report [24]. An appropriately designed study is needed to clarify the above important relationship of the severity of xerostomia to the development of candidiasis.

The high overall prevalence of ulcerative/pseudomembranous mucositis grade 2, 3 and 4 during and at the end of RT ( $82 \%$ and $77.7 \%$ respectively) agrees with the high prevalence of oral mucositis reported previously $[3,5,6,22-24$, 26]. The above unchanged prevalence of mucositis during and at the end of RT further verifies, in the clinical setting, that mucositis is not an infectious process. Infection, bacterial, fungal, and viral, is a secondary phenomenon, which may exacerbate mucositis $[11,12]$. The mechanisms with which infections may exacerbate mucositis have not yet been delineated.

In relation to bacterial infections bacterial colonization may occur once mucositis-associated ulceration develops. Bacteria and their metabolites are likely to intensify the inflammatory process of mucositis. A full delineation of their role in exacerbating the oral mucositis, however, remains unclear [17-20].

Table 3. Patients' Follow up by RT Interruption Status

\begin{tabular}{|c|c|c|c|c|c|}
\hline PARAMETER & \multicolumn{2}{|c|}{ Patients without RT Interruption } & \multicolumn{2}{|c|}{ Patients with RT Interruption } & \multirow{2}{*}{ p-value Pearson's $X^{2}$} \\
\hline Follow up & $\mathbf{N}=\mathbf{1 1 7}$ & $\%$ & $\mathrm{~N}=\mathbf{1 8}$ & $\%$ & \\
\hline Alive, free of disease & 60 & 51.2 & 7 & 41.2 & \multirow{4}{*}{ NS } \\
\hline Alive, with disease & 16 & 13.6 & 1 & 5.8 & \\
\hline Died & 22 & 18.8 & 2 & 11.8 & \\
\hline Lost of follow up & 19 & 16.2 & 8 & 44.4 & \\
\hline
\end{tabular}

NS: not statistically significant $\mathrm{p}>0.05$ 
Candidiasis, on the other hand, has been described in head and neck RT, often superimposed on mucositis, with an incidence of $27 \%$ to $50 \%$ [21-26], being developed in Candida carriers. During head and neck RT, $57 \%$ to $70.6 \%$ of the patients have been documented as Candida carriers $[22,23,25,27,28]$.

The antifungal treatment administered to $70.3 \%$ of the patients is comparable to the reported $57 \%$ to $70.6 \%$ prevalence of Candida carriage, both in the literature [25, 27, 28] and in our patient cohorts [22, 23]. Thus, there is concurrence between the presumed, clinical infection and the laboratory-verified Candida carriage.

Despite the well documented high prevalence of Candida carriage in head and neck cancer patients who receive RT and as reported in our previous studies [22, 23] lack of the assessment of the fungal status, before and after the antifungal medication, due to the daily clinical practice issues, remains a weakness of the present study.

Also and again due to daily clinical practice issues, cytology smear was taken from only 46 of all 96 patients $(47.9 \%)$, who had developed ulcerations, with a clinical suspicion for herpes and who had received antivirals. Fourteen of the 46 smears $(30.4 \%)$ were found positive for herpetic infection. The viral cytology laboratory verification of 46 of all 96 patients (47.9\%), who developed ulcerations, clinically suspicious for herpes, did not permit exact determination of overall incidence of herpetic infection complicating the ulcerations of mucositis, representing another weakness of the present study. Collection of oral mucosal specimens from painful ulcerative oral mucositis can be difficult if the patient is already experiencing moderate-severe oral mucosal pain.

The $71 \%$ (96 of 135) of patients, who received antivirals, is higher than the $29 \%$ prevalence of herpetic infection verified previously in our Clinic [23] and the $30.4 \%$ of positive smears documented in the present study. This denotes an over-treatment and an over-diagnosis in terms of the clinical setting, of herpetic infection, obviously related to the difficulty/inability to clinically differentiate the ulcers of mucositis grade 2, 3, and 4 from the ulcers of herpetic infection in head and neck cancer radiotherapy.

The non-significant differences in symptoms between the patients who received RT alone as opposed to the patients who received concomitant chemotherapy has also been noted in our previous studies $[23,24]$ and in a recent prospective multicenter study [5]. These findings denote that chemotherapy, administered concurrently with RT, may not play a significant role in radiation-associated symptom burden. In contrast, in other studies, chemoradiotherapy has been shown to be related to an increased risk of mucositis $[6,8]$. Factors such as systemic condition of the patients or the type of chemotherapy, the mode of data collection, prospectively or retrospectively, and other factors may play a role in the above controversy.

The insignificant differences in the survival and prognosis of the patients with and without RT interruptions in the present cohort can not be further evaluated, due to the study timeframe.
The advantage of this study is the prospective evaluation of mucosal toxicity in an ambulatory-based clinical dental practice using a specific scale for scoring oral mucositis.

In conclusion, the significant reduction of the prevalence of severe oral mucositis, pain and xerostomia, at the end of $\mathrm{RT}$, after the treatment and prevention of fungal and viral infections, denotes an important role of candidiasis and herpes in the severity of these complications during head and neck radiotherapy, with or without chemotherapy. Limitations of the study are the practical issues of the lack of the verification of the fungal status, before and after treatment and of the verification of the viral status in only $47.9 \%$ of the patients, with a clinical suspicion for herpes.

The present study is important because it presents the data outside the context of a clinical trial, it agrees with previous studies and it further emphasizes the need to investigate and clarify the role of antifungal and antiviral prophylaxis in the severity of oral mucositis, pain and xerostomia in head and neck cancer patients who receive RT with or without chemotherapy.

\section{ACKNOWLEDGEMENTS}

We thank Professor Douglas E. Peterson for his valuable editorial input during the writing of the manuscript.

The work was partially supported by the National and Kapodistrian University, Special Research Account, Code Number 70/4/8884.

Secretarial assistance was provided by the Hellenic Association of Supportive Care of the Oral Cavity in Cancer - HASCOCC.

\section{REFERENCES}

[1] Spijkervet FKL, Van Saene HKF, Panders AK, Vermey A, Mehta DM. Scoring irradiation mucositis in head and neck cancer patients. J Oral Pathol Med 1989; 18: 167-71.

[2] Ohrn KEO, Wahlin YB, Sjoden PO. Oral status during radiotherapy and chemotherapy: a descriptive study of patient experiences and the occurrence of oral complications. Support Care Cancer 2001; 9: 247-57.

[3] Trotti A, Bellm LA, Epstein JB, et al. Mucositis incidence, severity and associated outcomes in patients with head and neck cancer receiving radiotherapy with or without chemotherapy: a systematic literature review. Radiother Oncol 2003; 66: 253-62.

[4] Epstein JB, Beaumont JL, Gwede CK, et al. Longitudinal evaluation of the oral mucositis weekly questionnaire-head and neck cancer, a patient-reported outcomes questionnaire. Cancer 2007; 109: 1914-22.

[5] Elting LS, Keefe DM, Sonis ST, et al. Patient-reported measurements of oral mucositis in head and neck cancer patients treated with radiotherapy with or without chemotherapy. Cancer 2008; 113: 2704-13.

[6] Elting LS, Cooksley CD, Chambers MS, Garden AS. Risk, outcomes, and costs of radiation-induced oral mucositis among patients with head-and-neck malignancies. Int J Radiat Oncol Biol Phys 2007; 68: 1110-20.

[7] Nonzee NJ, Dandade NA, Markossiant T, et al. Evaluating the supportive care costs of severe radiochemotherapy-induced mucositis and pharyngitis. Cancer 2008; 113: 1446-52.

[8] Vera-Llonch M, Oster G, Hagiwara M, Sonis ST. Oral mucositis in patients undergoing radiation treatment for head and neck carcinoma. Risk factors and clinical consequences. Cancer 2006; 106: 329-36. 
[9] Rosenthal DI. Consequences of mucositis-induced treatment breaks and dose reductions on head and neck cancer treatment outcomes. Support Oncol 2007; 5: 23-31.

[10] Russo G, Haddad R, Posner M, Machtay M. Radiation treatment breaks and ulcerative mucositis in head and neck cancer. The Oncologist 2008; 13: 886-98.

[11] Sonis ST, Elting LS, Keefe D, et al. Perspectives on cancer therapy-induced mucosal injury. Pathogenesis, measurement, epidemiology, and consequences for patients. Cancer Suppl 2004; 100: 1995-2025.

[12] Sonis ST. Pathobiology of oral mucositis: Novel insights and opportunities. Support Oncol 2007; 5: 3-11.

[13] Keefe DM, Schubert MM, Elting LS, et al. Updated clinical practice guidelines for the prevention and treatment of mucositis. Cancer 2007; 109: 820-31.

[14] Silverman SJr. Diagnosis and management of oral mucositis. Support Oncol 2007; 5: 13-21.

[15] Teister N, Sonis S. Mucositis: biology and management. Curr Opin Otolaryngol Head Neck Surg 2007; 15: 123-9.

[16] Lalla RV, Sonis ST, Peterson DE. Management of oral mucositis in patients with cancer. Dent Clin North Am 2008; 52: 61-77, viii.

[17] Spijkervet FKL, Van Saene HKF, Van Saene JJM, et al. Effect of selective elimination of the oral flora on mucositis in irradiated head and neck cancer patients. J Surg Oncol 1991; 46: 167-73.

[18] El-Sayed S, Nabid A, Shelley W, et al. Prophylaxis of radiationassociated mucositis in conventionally treated patients with head and neck cancer: A double- blind, phase III, randomized, controlled trial evaluating the clinical efficacy of an antimicrobial lozenge using a validated mucositis scoring system. J Clin Oncol 2002; 20: 3956-63.

[19] Donelly JP, Bellm LA, Epstein JB, Sonis ST, Symonds RP. Antimicrobial therapy to prevent or treat oral mucositis. Lancet Infect Dis 2003; 3: 405-12.

[20] Barasch A, Elad S, Altman A, Damato K, Epstein JB. Antimicrobials, mucosal coating agents, anesthetics, and nutritional supplements for alimentary tract mucositis. Support Care Cancer 2006; 14: 528-32.

[21] Epstein JB, Freilich MM, Le ND. Risk factors for oropharyngeal candidiasis in patients who receive radiation therapy for malignant conditions of the head and neck. Oral Surg Oral Med Oral Pathol 1993; 76: 169-74.

[22] Nicolatou-Galitis O, Velegraki A, Sotiropoulou-Lontou A, et al. Effect of fluconazole antifungal prophylaxis on oral mucositis in head and neck cancer patients receiving radiotherapy. Support Care Cancer 2006a; 14: 44-51.

[23] Nicolatou-Galitis O, Athanassiadou P, Kouloulias V, et al. Herpes simplex virus-1 (HSV-1) infection in radiaton-induced oral mucositis. Support Care Cancer 2006b; 14: 753-62.

[24] Nicolatou-Galitis O, Dardoufas K, Markoulatos P, et al. Oral pseudomembranous candidiasis, herpes simplex virus-1 infection, and oral mucositis in head and neck cancer patients receiving radiotherapy and granulocyte-macrophage colony-stimulating factor (GM-CSF) mouthwash. J Oral Pathol Med 2001; 30: 471-80.

[25] Dahiya MC, Redding SW, Dahiya RS, et al. Oropharyngeal candidiasis caused by non-albicans yeast in patients receiving external beam radiotherapy for head-and-neck cancer. Int $\mathbf{J}$ Radiat Oncol Biol Phys 2003; 57: 79-83.

[26] Nicolatou-Galitis O, Sotiropoulou-Lontou A, Velegraki A, et al. Oral candidiasis in head and neck cancer patients receiving radiotherapy, with amifostine cytoprotection. Oral Oncol 2003; 39: 397-401.

[27] Jham BC, Franca EC, Olivera RR, et al. Candida oral colonization and infection in Brazilian patients undergoing head and neck radiotherapy: a pilot study. Oral Surg Oral Med Oral Pathol Oral Radiol Endod 2007; 103: 355-58.

[28] Paula CR, Sampaio MCC, Birman E, Siqueira AM. Oral yeasts in patients with cancer of the mouth, before and during radiotherapy. Mycopathologia 1990; 112: 119-24.

[29] Mucke R, Kaben U, Libera T, et al. Fluconazole prophylaxis in patients with head and neck tumours undergoing radiation and radiochemotherapy. Mycoses 1998; 41: 421-3.

[30] Koc M, Aktas E. Prophylactic treatment of mycotic mucositis in radiotherapy of patients with head and neck cancers. Jpn J Clin Oncol 2003; 33: 57-60.

[31] Djuric M, Jankovic L, Jovanovic T, et al. Prevalence of oral herpes simplex virus reactivation in cancer patients: a comparison of different techniques of viral detection. J Oral Pathol Med 2009; 38: 167-73.

[32] Cox JD, Stetz J, Pajak TF. Toxicity criteria of the Radiation Therapy Oncology Group (RTOG) and the European Organization for Research and Treatment of Cancer (EORTC). Int J Radiat Oncol Biol Phys 1995; 31: 1341-6.

[33] Nicolatou-Galitis O, Sarri T, Dardoufas K, et al. Oral mucositis, pain and xerostomia in patients with head and neck cancer who receive chemoradiotherapy with or without cetuximab. Open Clin Cancer J 2010; 4: 6-14.

Received: March 23, 2010

Revised: August 01, 2010

Accepted: November 11, 2010

(c) Nicolatou-Galitis et al.; Licensee Bentham Open.

This is an open access article licensed under the terms of the Creative Commons Attribution Non-Commercial License (http://creativecommons.org/licenses/by$\mathrm{nc} / 3.0 /$ ), which permits unrestricted, non-commercial use, distribution and reproduction in any medium, provided the work is properly cited. 